\title{
Research on Emerging Infections Offers an Opportunity for Public Health Intelligence on Non-Communicable Diseases: Hypertension Prevalence in Volunteers for an Ebola Vaccine Trial in Northern Sierra Leone ${ }^{+}$
}

\author{
Dickens Kowuor 1,2, David Ishola 1,2, , Muhammed Afolabi 1,2, Frank Baiden 1,2, \\ Kwabena Owusu-Kyei 1,2, Baimba Rogers 2,3, Osman Bah 2,3, Ibrahim Swaray 2,3, \\ Joseph Edem-Hotah ${ }^{3}$, Gibrilla Fadlu Deen ${ }^{3}$, Daniela Manno ${ }^{1}$, Philip Ayieko ${ }^{1}$, \\ Julie Foster 1, Pauline Akoo 1,2, Daniel Tindanbil 1,2, Mohamed Samai ${ }^{3}$, \\ Brian Greenwood ${ }^{1}$, Bailah Leigh ${ }^{2,3}$ and Deborah Watson-Jones ${ }^{1}$ \\ 1 London School of Hygiene and Tropical Medicine, London WC1E 7HT, UK; \\ dickens.kowuor@lshtm.ac.uk (D.K.); muhammed.afolabi@lshtm.ac.uk (M.A.); \\ frank.baiden@lshtm.ac.uk (F.B.); kwabena.owusu-kyei@lshtm.ac.uk (K.O.-K.); \\ Daniela.manno@lshtm.ac.uk (D.M.); philip.ayieko@lshtm.ac.uk (P.Ayieko); julie.foster@lshtm.ac.uk (J.F.); \\ Pauline.akoo@lshtm.ac.uk (P.Akoo); Daniel.tindanbil@lshtm.ac.uk (D.T.); \\ brian.greenwood@lshtm.ac.uk (B.G.); deborah.watson-jones@lshtm.ac.uk (D.W.-J.) \\ 2 EBOVAC1 Study Site, Kambia District 47235, Sierra Leone; baimba.rogers@usl.edu.sl (B.R.); \\ osman.bah@usl.edu.sl (O.B.); ibrahim.swaray@usl.edu.sl (I.S.); bailah.leigh@usl.edu.sl (B.L.) \\ 3 College of Medicine and Allied Health Sciences (COMAHS), University of Sierra Leone, \\ Freetown PMB 595, Sierra Leone; joseph.hotah@usl.edu.sl (J.E.-H.); gibrilla.deen@usl.edu.sl (G.F.D.); \\ Mohamed.samai@usl.edu.sl (M.S.) \\ * Correspondence: david.ishola@lshtm.ac.uk \\ + Presented at the 5th African Conference on Emerging Infectious Diseases, Abuja, Nigeria, 7-9 August 2019. \\ Published: 25 May 2020
}

\begin{abstract}
Introduction: The West African Ebola outbreak of 2014-2016 necessitated clinical trials in communities with limited health data. The EBOVAC-Salone Ebola vaccine trial is ongoing in the largely rural Kambia District in northern Sierra Leone. To gain a baseline insight into our local noncommunicable disease (NCD) epidemiology, we examined screening blood pressure (BP) measurements in trial volunteers. Methods: $\mathrm{BP}$ involved taking multiple readings using an Omron M6 sphygmomanometer in rested individuals. We classified BP by the European 2018 ESC/ESH guidelines: optimal BP, normal or high-normal BP, or hypertension (systolic $\geq 140 \mathrm{mmHg} \pm$ diastolic $\geq 90 \mathrm{mmHg}$ ) with Grade 1, 2, or 3 (G1HT, G2HT, G3HT) severity levels. Results: Of 870 volunteers, $220(25.3 \%)$ had optimal BP, $236(27.13 \%)$ had normal BP, and $250(28.7 \%)$ had high-normal BP. The remaining $164(18.9 \%)$ were hypertensive. By gender, 16.5\% (109/668) of males and 27.2\% (55/202) of females were hypertensive. Among hypertensives, $62.2 \%$ had G1HT, $18.3 \%$ had G2HT, and $19.5 \%$ had G3HT. Twenty-two (13.4\%) were previously diagnosed, with eight on treatment. Forty-one had isolated systolic hypertension. The prevalence significantly increased with age $(p<0.0001)$, with $5.3 \%(27 / 514)$ in the age-category $18-29 \mathrm{y}, 18.6 \%(29 / 156)$ in $30-39 \mathrm{y}, 49.4 \%(84 / 170)$ in $40-59 \mathrm{y}$, and $80 \%(24 / 30)$ in $\geq 60 \mathrm{y}$. The severity also increased with age, with $54.9 \%$ of G1HT, $76.7 \%$ of G2HT, and $90.7 \%$ of G3HT being aged $\geq 40 \mathrm{y}$. In total, $36.6 \%$ (60/164) of hypertensives were overweight or obese. Discussion: In an economically disadvantaged, Ebola-affected rural West African community where NCD might not traditionally be thought prevalent, almost one in five adults were found to be hypertensive and were mostly unaware. Additionally, nearly one in three had high-normal BP.
\end{abstract}


Together, these findings portend a potent, largely silent, and potentially growing NCD threat, and illustrate that infectious disease (ID) studies could provide opportunities for pragmatic NCD data. As both ID and NCD are putatively promoted by overlapping pro-inflammatory and povertydriven factors, a cross-paradigmatic "multiplex" approach, whereby ID studies prospectively incorporate NCD-related sub-studies (and vice versa), might optimize limited research resources for enhanced public health benefit.

Keywords: Sierra Leone; Ebola; non-communicable disease; hypertension; West Africa; clinical trial; participant screening; Africa; emerging infectious diseases. 